\title{
ON EXTENSIONS OF CAYLEY ALGEBRAS
}

\section{MARGARET M. HUMM AND ERWIN KLEINFELD ${ }^{1}$}

Kaplansky in Theorem 2 of [3] has shown that if $A$ is an alternative algebra with identity element 1 which contains a subalgebra $B$ isomorphic to a Cayley algebra and if 1 is contained in $B$ then $A$ is isomorphic to the Kronecker product $B \otimes T$, where $T$ is the center of A. Jacobson in Theorem 2 of [2] has shown that if $A$ is an alternative algebra which contains a subalgebra $B$ isomorphic to a Cayley algebra, then the identity $e$ of $B$ must lie in the center of $A$, provided $A$ has characteristic different from 2 . He also has given a new proof of the Kaplansky result, using his classification of completely reducible alternative bimodules. In the present note we present a generalization of the aforesaid result by Jacobson, which incidentally is also valid for characteristic 2 .

THEOREM. Let $A$ be an alternative algebra over $F$ and $B$ any subalgebra with identity $e$. Then consider the following two conditions.

(i) There exist $x, y$ in $B, \alpha$ in $F$ such that $e=\alpha(x, y)^{4}$, where $(x, y)$ $=x y-y x$.

(ii) The ideal $I$ of $B$, generated by all associators of $B$ equals $B$. If $B$ satisfies (i) then e must be in the nucleus $N$ of $A$. If $B$ satisfies (i) and (ii) then e must be in the center $C$ of $A$.

Proof. It will be helpful to recall some identities that hold in all alternative rings $R$. Let $p, q, r, s, t, x, y, z$ be arbitrary elements of $R$ and $n$ an arbitrary element of the nucleus $N^{\prime}$ of $R$. Then

(1) $(s, t)^{4}$ is in $N^{\prime}$,

(2) $(n, r)$ is in $N^{\prime}$,

(3) $(n,(x, y, z))=0$,

(4) $(n, r)(x, y, z)=-(n, x)(r, y, z)$,

(5) $\left(p^{2}, q\right)=p(p, q)+(p, q) p$.

A proof of (1) may be found in Theorem 3.1 (ii) of [5]. Proofs of (2), (3) and (4) are contained in Lemma 2.3 (ii), (iii) and (iv) of [4]. Identity (5) may be verified directly by expanding both sides of the equation and using the alternative law. If $B$ satisfies the hypothesis and condition (i), then one may apply (1) directly to obtain that $e$

Received by the editors September 18, 1965 and, in revised form, February 23, 1966.

1 This paper was supported in part by a grant from U. S. Army Research (Durham) to Syracuse University. 
belongs to $N$. If $B$ also satisfies condition (ii), then select $n=e, r$ as arbitrary in $A$ and $x, y, z$ arbitrary in $B$ and substitute this in (4). Then $(e, r)(x, y, z)=-(e, x)(r, y, z)=0$, since $(e, x)=0$. The associator ideal $I$ of $B$ may be characterized as the additive subgroup of $B$ generated by all elements of the form $(B, B, B)$ and $(B, B, B) B$. We have already proved that $(e, r)(B, B, B)=0$. But $(e, r)$ belongs to $N$ as a result of (2), so that $(e, r) \cdot(B, B, B) B=0$ is also obvious and hence $(e, r) I=0$. Since $I=B$ and $e$ itself belongs to $B$, we have $(e, r) e=0$. Using (2) we may substitute $n=(e, r)$ in (3) to obtain also that $(B, B, B)(e, r)=0$. As $I$ may also be characterized as the additive subgroup generated by elements of the form $(B, B, B)$ and $B(B, B, B)$, we obtain $I(e, r)=0$, and hence $e(e, r)=0$. At this point we substitute $p=e, q=r$ in (5) and obtain $(e, r)=\left(e^{2}, r\right)=e(e, r)$ $+(e, r) e=0$. This places $e$ in $C$ and the proof of the theorem is complete.

Condition (i) certainly holds when $B$ is taken to be a quaternion algebra and hence a priori if $B$ is a Cayley algebra. Since Cayley algebras are simple and not associative, condition (ii) clearly holds when $B$ is taken to be a Cayley algebra. Thus we obtain Jacobson's result as a corollary to our theorem. On the other hand one may readily construct other alternative algebras to which our theorem applies.

We conclude with an example that shows a quaternion algebra may be embedded as a subalgebra of an associative algebra and with the identity quaternion not in the center of the larger algebra. Consider the free associative algebra $S$ on the four generators $w, x, y, z$. Define relations on $x, y, z$ which make them behave as the quaternions $1, i, j$ respectively. In the quotient algebra $R$, words have the form

$$
q_{r} w^{k_{r}} \cdots q_{s} w^{k_{s}} \cdots
$$

where $q_{i}= \pm x, \pm y, \pm z, \pm y z$. Then $R$ contains a copy of the quaternions with identity $x$, but $w x \neq x w$, so that $x$ is not in the center of $R$. If an example that is alternative but not associative is desired, then one may take a direct product of $R$ with a Cayley algebra.

\section{BiBLIOGRAPHY}

1. A. A. Albert, On simple alternative rings, Canad. J. Math. 4 (1952), 129-135.

2. N. Jacobson, A Kronecker factorization theorem for Cayley algebras and the exceptional simple Jordan algebra, Amer. J. Math. 76 (1954), 447-452.

3. Irving Kaplansky, Semi-simple alternative rings, Portugal. Math. 10 (1951), $37-50$.

4. Erwin Kleinfeld, An extension of the theorem on alternative division rings, Proc. Amer. Math. Soc. 3 (1952), 348-351.

5. - Simple alternative rings, Ann. of Math. 58 (1953), 544-547. 
6. - A characterization of the Cayley numbers, Math. Assoc. America Studies in Mathematics, Vol. 2, pp. 126-143, Prentice-Hall, Englewood Cliffs, N. J., 1963.

Syracuse UNIVERSITY

\section{A CONDITION FOR A FINITE GROUP TO BE NILPOTENT}

\section{STEPHEN MONTAGUE AND GOMER THOMAS}

Let $\mathcal{H C}$ be a class of groups such that:

(i) If $G$ is in $\Re$, then every homomorphic image of $G$ is in $\Re$.

(ii) If $G$ is finite and $G / \phi(G)$ is in $\mathcal{H}$, where $\phi(G)$ is the Frattini subgroup of $G$, then $G$ is in $\mathfrak{H C}$.

Examples of such classes are the class of nilpotent groups and the class of supersolvable groups. Others can be found in a paper by Baer [1].

In this note a theorem of $\mathrm{P}$. Hall on nilpotent groups is proved as a corollary to the following:

THEOREM. If $G$ is a finite group with a subgroup $H$ such that $\phi(H)$ is normal in $G$ and $G / \phi(H)$ is in $\mathfrak{H C}$, then $G$ is in $\mathfrak{H C}$.

LEMMA (HUPPERT). Let $G$ be a finite group, $H$ be a subgroup of $G$, and $N$ be a subgroup of $H$ such that $N$ is normal in $G$ and $N \leqq \phi(H)$. Then $N \leqq \phi(G)$.

Proof. If not, $G$ would have to have a maximal subgroup $U$ such that $N \ddagger U$. Then $H=G \cap H=N U \cap H=N(U \cap H)=U \cap H$, since $N \leqq \phi(H)$. But this implies $H \leqq U$, contrary to $N \leqq U$.

Proof OF THEOREM. An application of the Lemma with $N=\phi(H)$ shows that $\phi(H) \leqq \phi(G)$. Hence $G / \phi(G)$ is in $\mathcal{F}$, and so $G$ is in $\mathcal{H}$.

CoRollary. If $G$ is a finite group with a normal subgroup $H$ such that $H$ is nilpotent and $G / H^{\prime}$ is nilpotent, where $H^{\prime}$ is the commutator subgroup of $H$, then $G$ is nilpotent.

Proof. Since $H$ is nilpotent, $\phi(H)$ contains $H^{\prime}$. Hence $G / \phi(H)$ is

Received by the editors September 22, 1965. 